Planetary Systems in the Universe - Observation, Formation and Evolution Proceedings IAU Symposium No. 202, (C)2004 IAU

Alan Penny, Pawel Artymowicz, Anne-Marie Lagrange, \& Sara Russell, eds.

\title{
First attempt in searching for HD209458b's exosphere
}

\author{
${ }^{1}$ Claire Moutou, ${ }^{2}$ Athena Coustenis, ${ }^{2}$ Jean Schneider, \\ ${ }^{2}$ Richard St Gilles, ${ }^{3}$ Michel Mayor, ${ }^{3}$ Didier Queloz and ${ }^{1}$ Andreas Kaufer \\ ${ }^{1}$ ESO, Casilla 19001, Santiago, Chile \\ ${ }^{2}$ Observatoire de Paris-Meudon, 5 Place Jules Janssen \\ ${ }^{3}$ Geneva Observatory, 51 ch. des Maillettes, 1290 Sauverny, Switzerland
}

\begin{abstract}
The transit of the planet orbiting HD209458 has been observed using VLT/UVES, in search for faint signatures of an extended envelope of gas possibly surrounding the planet, called exosphere. Preliminary results are shown and described. They show no evidence so far of exospheric signatures.
\end{abstract}

\section{Introduction}

The atmosphere of "hot Jupiters" are expected to escape into an extended envelope of matter called exosphere (Chassefière 1996, Schneider et al. 1998). Neutral and ionized species should be present around the planet up to distances of $\simeq 10$ planet radii (Coustenis et al. 1998). Interactions with the stellar wind at such short distances result in a exosphere structure comparable to a cometary tail (Fig 1). The tail geometry depends on the stellar wind velocity, escape velocity of ionic species and on the orbital motion of the planet.

Detection of the exosphere of an extra-solar planet by spectroscopy would bring a huge breakthrough in the field, allowing the identification and abundance measurement of its atmospheric species. Constraints on atmospheric models (Seager $\&$ Sasselov 2000) would be accurately refined. Previous attempts on 51 Peg were not succesful so far (Coustenis et al. 1997; Rauer et al. 2000). The recent discovery of the transiting system HD209458 (Charbonneau et al 2000; Henry et al. 2000; Mazeh et al. 2000) brings ideal conditions for such a search. We have observed HD209458 during a transit of its nearby planet $\left(0.69 \mathrm{M}_{\mathrm{Jup}}, 3.5\right.$ day period) on Dec. 17th with UVES, the UV-visible echelle spectrograph on Kueyen (VLT 2).

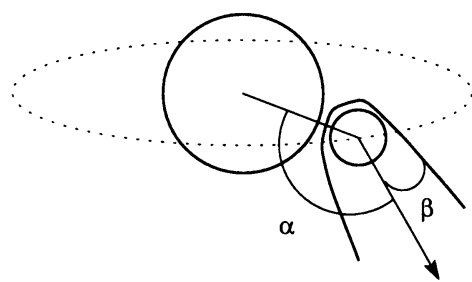

Figure 1. Scheme of the tail-like exosphere around the planet. 


\section{Observations and Strategy}

Table 1 displays observational parameters; we took 3 spectra during transit and 3 spectra one night later, for comparison. The idea is to search for additional absorption features on the on-transit spectrum, which would be due to the planet gaseous extension. The signature should be detected at $3 \sigma$ on each individual spectrum which allows to distinguish from numerous noise features. UVES was used in Dichroic $1390+564$ setting, i.e., we covered the ranges $328-456.2 \mathrm{~nm}$ ("B"), 458.3 - $564.4 \mathrm{~nm}$ ("RL") and $565.4-668.6 \mathrm{~nm}$ ("RU"), at a spectral resolution of about 60,000 . The achieved signal-to-noise ratio on the combined spectrum is, respectively for each detector, 320,520 and 780 . Reference stars were also observed. The combined spectrum is shown on Fig. 2.

Table 1. Table of exposures: orbital phase, integration time, achieved SNR per arm, airmass and external seeing at airmass $=1$.

\begin{tabular}{llllll}
\hline Exposure & $\begin{array}{l}\text { orbital } \\
\text { phase }\end{array}$ & $\begin{array}{l}\text { integ. } \\
\text { time }(\mathrm{sec})\end{array}$ & $\begin{array}{l}\text { estim. SNR } \\
\text { (B/RL/RU) }\end{array}$ & Airmass & $\begin{array}{l}\text { Seeing } \\
\text { (arcsec) }\end{array}$ \\
\hline HD209458-ON 1 & 0.000540 & 600 & $160 / 230 / 340$ & 2.16 & 0.90 \\
HD209458-ON 2 & 0.00264 & 900 & $210 / 340 / 510$ & 2.31 & 0.90 \\
HD209458-ON 3 & 0.00610 & 700 & $180 / 310 / 470$ & 2.61 & 0.80 \\
\hline HD209458-OFF 1 & 0.28520 & 600 & $200 / 330 / 470$ & 2.27 & 0.55 \\
HD209458-OFF 2 & 0.28732 & 540 & $190 / 310 / 460$ & 2.45 & 0.60 \\
HD209458-OFF 3 & 0.28925 & 600 & $180 / 300 / 450$ & 2.64 & 0.58 \\
\hline
\end{tabular}

The careful inspection of the "on" /"off" ratio spectrum, after proper correction of the residual Doppler shift, shows a few features. Most of them can be identified as instrumental effects, e.g. residual of cosmic rays, black spots on the CDD, or to slightly differential airmasses which result in intensity variations of the telluric lines. An absorption feature at $614.68 \mathrm{~nm}$ seems to be the unique unexplained signature: it is present in all "on" spectra and weaker in all "off" spectra; it matches one signature of $\mathrm{H}_{2} \mathrm{O}^{+}$, one of the main molecular species expected in such an environment. But 1) other lines of the same species are not detected and 2) the spectrum of one reference star also shows an absorption at $614.68 \mathrm{~nm}$, which both argue against a real identification of an exospheric signature. Another doubt subsists for NaI doublet absorption, for which the planet contribution is blended by stellar and terrestrial features.

\section{Conclusions}

The results are discussed more extensively in a coming paper (Moutou et al. 2000). Here we draw the global conclusions:

- No significant signature of an exosphere is detected on the UVES spectrum, at a level of approximately 2 to $3 \%$. This detection limit is however above the predicted depth of the signatures, so that we cannot derive upper limits of species' abundances.

- The low airmass and the too short time coverage of the event did not allow to reach the detection limit of $\simeq 1 \%$ deep absorption lines on top of the star 


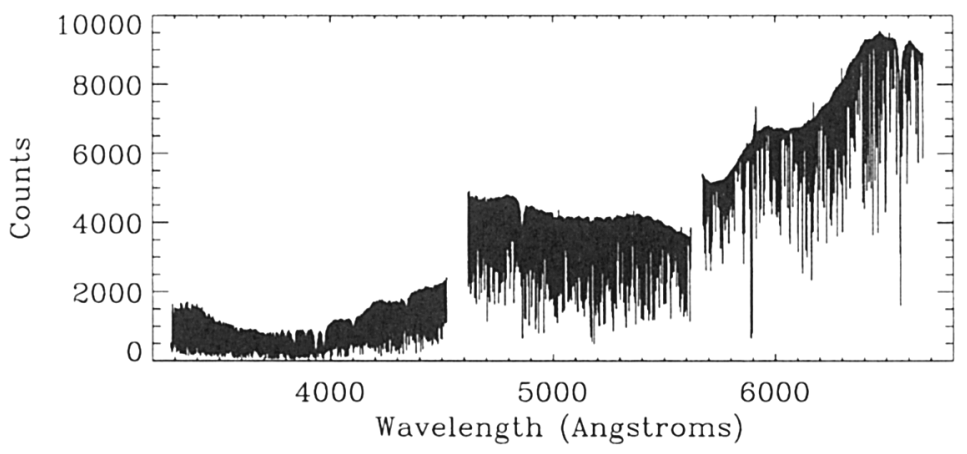

Figure 2. The complete spectrum obtained with UVES during HD209458b's transit. This is a combination of three exposures, after reduction and optimal extraction.

spectrum.

- Marginal detections at $614.68 \mathrm{~nm}$ and close to the stellar NaI doublet are seen. Their confirmation requires further observations.

- A more complete set of observations is under analysis: benefiting a longer time coverage, we should then be able to constrain the chemistry of HD209458b's exosphere, and derive the tail geometry from absorption variability during the transit (Coustenis et al. in prep.).

\section{References}

Charbonneau D., Brown T., Latham D., \& Mayor M. 2000a, ApJ, 529, L45

Chassefière E. 1996, J. Geophys. Res., 101, 26039

Coustenis A. et al. 1997, in "Planets beyond the Solar System and the Next Generation of Space Missions", eds. Soderblom, ASP, 119, 101

Coustenis A. et al. 1998, in "Brown dwarfs and Extrasolar planets", ASP 134, eds. Rebolo et al., 296

Guillot T. et al. 1996, ApJ, 459, L35

Henry G., Marcy G. Butler R., Vogt S., 2000, ApJ, 529, L41

Mazeh T. et al. 2000, ApJ, 532, L55

Moutou C. et al 2000, submitted to A\&A

Rauer H. et al. 2000a, A\&A, 355, 573

Schneider J. et al. 1998, in "Brown Dwarfs and Extrasolar Planets", eds. Rebolo et al., ASP 134, p.241

Seager S. \& Sasselov D. 2000, ApJ, 537, 916 